\title{
A Machine Learning Approach for Near-Fall Detection based on Inertial and Force Data while using a Conventional Rollator
}

\author{
Nuno Ferrete Ribeiro, Ana Pereira, Joana Figueiredo, José A. Afonso and Cristina P. Santos
}

\begin{abstract}
Falls are a major concern for society. They may result in death or in several injuries that require motor assistance, representing an economic burden. To overcome these problems, a diversity of fall prevention strategies implemented on assistive devices such as smart walkers, have been widely explored. This study presents a novel strategy by using exclusively information from wearable sensors to detect near-falls while the subject uses a conventional rollator. A comparative analysis was performed to identify the most suitable classifier and the most relevant subset of features for detecting near-fall events. Ten able-bodied subjects performed 240 trials and simulated 180 near-falls with the rollator. The Ensemble Learning with the first 51 ranked features by the mRMR presented the best performance results (Accuracy $\mathbf{= 9 5 . 1 8 \%}$; Detection time before recovery= $1.48 \pm 0.68 \mathrm{~s}$ ). The results show that this strategy is suitable for use with conventional rollators, which are more used than smart walkers.
\end{abstract}

\section{INTRODUCTION}

WHEN smart walkers detect situations that may lead to a fall, they usually stop providing support to the user [1]. Current fall prevention strategies usually require information from non-wearable sensors placed on the walker, e.g. laser range finders (LRF) [2], stereo cameras [3], force/torque sensors [2], and depth cameras [4]. Smart walkers are less used than conventional rollators. However, the number of near-fall detection strategies for conventional rollators is lower. To the best knowledge of the authors, there is yet no near-fall detection strategy completely independent from the rollator and based only on an Inertial Measurement Unit (IMU) placed at the user's lower trunk and Force Sensitive Resistors (FSRs) in the feet. These sensors have already been studied as mentioned in [5] but without the support of any conventional rollator. This study aims therefore to implement the abovementioned strategy. A comparative analysis was performed to understand which is the most suitable machine learning classifier for near-fall detection, as well as the subset of most representative feature. This will allow to understand what information is really important for this classification problem towards the minimal sensor setup and computational load for real-time application.

This work has been supported by the FCT - Fundação para a Ciência e Tecnologia - with the scholarship reference PD/BD/141515/2018, by the FEDER funds through the Programa Operacional Regional do Norte and national funds from FCT with the SmartOs project under Grant NORTE01-0145-FEDER-030386, and under the national support to RD units grant, through the reference project UIDB/04436/2020 and UIDP/04436/2020.

N. F. Ribeiro, A. Pereira, J. Figueiredo, J. A. Afonso and C. P. Santos are with the Center for MicroEletroMechanical Systems (CMEMS), University of Minho, 4800-058, Guimarães, Portugal (corresponding author to provide e-mail: nuno.fribeiro@dei.uminho.pt).

\section{METHODS}

\section{A. System Overview and Experimental Protocol}

The proposed system (Fig. 1) comprises an IMU located near to L5 lumbar vertebra and S1 sacral vertebra where the Center of Mass (CoM) is usually considered; shoes equipped with four FSRs to detect foot contacts with the ground; and Arduino for sensor data acquisition at $100 \mathrm{~Hz}$. Trunk's acceleration and angular velocity were measured on three directions: Anteroposterior (AP), Vertical (V) and Mediolateral (ML). The experiments involved 10 able-bodied subjects (5 females and 5 males) with a mean age of $25.00 \pm 1.61$ years old, a mean height of $1.69 \pm 0.11 \mathrm{~m}$ and a mean weight of $66.50 \pm 11.32 \mathrm{~kg}$. All participants provided written and informed consent, respecting the ethical conduct defined by the University of Minho Ethics Committee. Participants were asked to walk with the support of a rollator for 4 different activities at 2 different gait speeds (comfortable and slow): walk forward for 10 meters; and walk forward and simulate near-falls to the right, left and forward. Each simulation was performed after an audible sound randomly applied by the assessor. Participants performed three trials per condition.

\section{B. Data Processing and Machine-Learning Approach}

We performed the following procedures after data collection: i) data processing using a 1st order lowpass filter (exponential smoothing) with 0.5 as the smoothing factor and a cut-off of $10 \mathrm{~Hz}$ [6]; ii) feature calculation which resulted in a data set with a total of 169 features (Table I); iii) feature normalization by subject's height and min-max scaling [0;1]; iv) feature selection by using two methods - MinimumRedundancy Maximum-Relevancy (mRMR) and Relief-F and adapting Principal Component Analysis (PCA); v) data labelling where near-falls only were considered after the last valid and regular step before the near-fall simulation; and vi) splitting data by using the hold-out method (70\% and 30\% for training and test, respectively). Subsequently, we implemented a 5-fold cross-validation (CV) with 10 repetitions using only training data to: i) select the best subset of features respecting the rankings; and ii) compare machine learning classifiers (Discriminant Analysis, K-Nearest Neighbors KNN, Ensemble Learning, Decision Tree - DT, and Support Vector Machine) using only the subset of features found previously. From these procedures, we identified the most accurate machine learning classifier for near-fall detection and conducted a hyperparameter optimization. Lastly, we implemented a post-processing method that uses the current and past classifier outputs to eliminate misclassifications. 


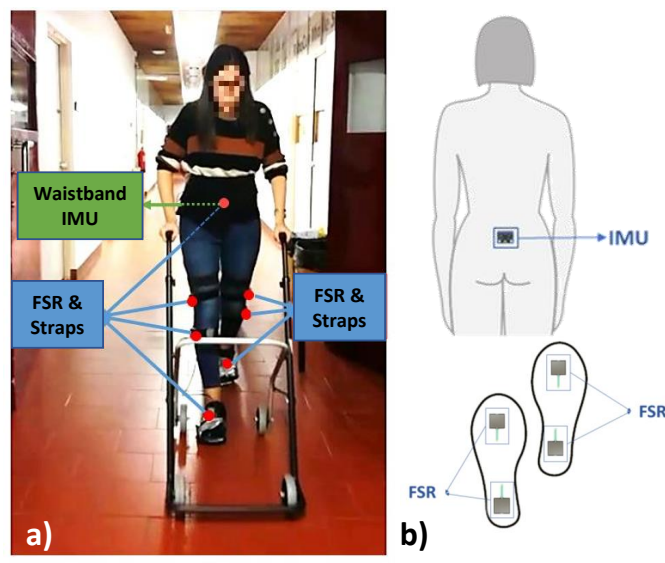

Fig. 1. System description: a) Waistband IMU and rollator; b) Wearable sensors location (IMU - back lower trunk; FSR - heel and toe).

\section{RESULTS AND DISCUSSION}

A total of 180 simulated near-falls using a conventional rollator were analyzed for near-fall detection. Based on 5fold $\mathrm{CV}$ results, the best performances were achieved with KNN, Ensemble Learning and DT with features ranked by mRMR and Relief-F. However, the KNN stands out with the following performance and with the first 59 ranked features by Relief-F: Accuracy $=99.93 \%$, Sensitivity $($ SENS $)=$ $99.71 \%$, Specificity $($ SPEC $)=99.97 \%$, Precision $($ PREC $)=$ $99.81 \%$, F1-score $=99.76 \%$ and Matthews correlation coefficient $(\mathrm{MCC})=99.71 \%$. The following classifiers appear below with an accuracy higher than 99\%: KNN (60 features - mRMR), Ensemble Learning (60 features - Relief-F), Ensemble Learning (51 features - mRMR) and DT (20 features - Relief-F). The classifiers were further tested with unseen data. The Ensemble Learning achieved the best performance using the first 51 features ranked by the mRMR (Table I): Accuracy $=95.18 \%, \mathrm{SENS}=71.63 \%, \mathrm{SPEC}=99.33 \%$, $\mathrm{PREC}=94.96 \%, \mathrm{~F} 1$-score $=81.66 \%$ and $\mathrm{MCC}=79.99 \%)$. The hyperparameter optimization outcomes are: i) Ensemble Aggregation Method - Bag; ii) Learning Cycles - 498; iii) Minimum Leaf Size - 1; and iv) No Learn Rate. Although accuracy and specificity values are high, the sensitivity value is low. Thus, we implemented a post-processing method based on a time window that contains the current and past classifier outputs to increase this value. A window size of 22 samples was obtained with training data. When using test data, a near-fall was detected on average $0.71 \pm 0.48 \mathrm{~s}$ after the start and $1.48 \pm 0.68 \mathrm{~s}$ before the recovery, being able to detect all 56 near-falls. The number of misclassified normal walking samples decreased $98 \%$. Although more detailed, our results are in line with the scientific literature [1]-[4].

\section{CONCLUSION}

We presented the Ensemble Learning with the first 51 features ranked by the mRMR from wearable data as an effective strategy for near-fall detection when walking with conventional walkers, advancing the state-of-the-art strategies that are focused on smart walkers less usually used by
TABLE I

FEATURE TABLE (51 FEATURES RANKED BY MRMR IN BOLD)

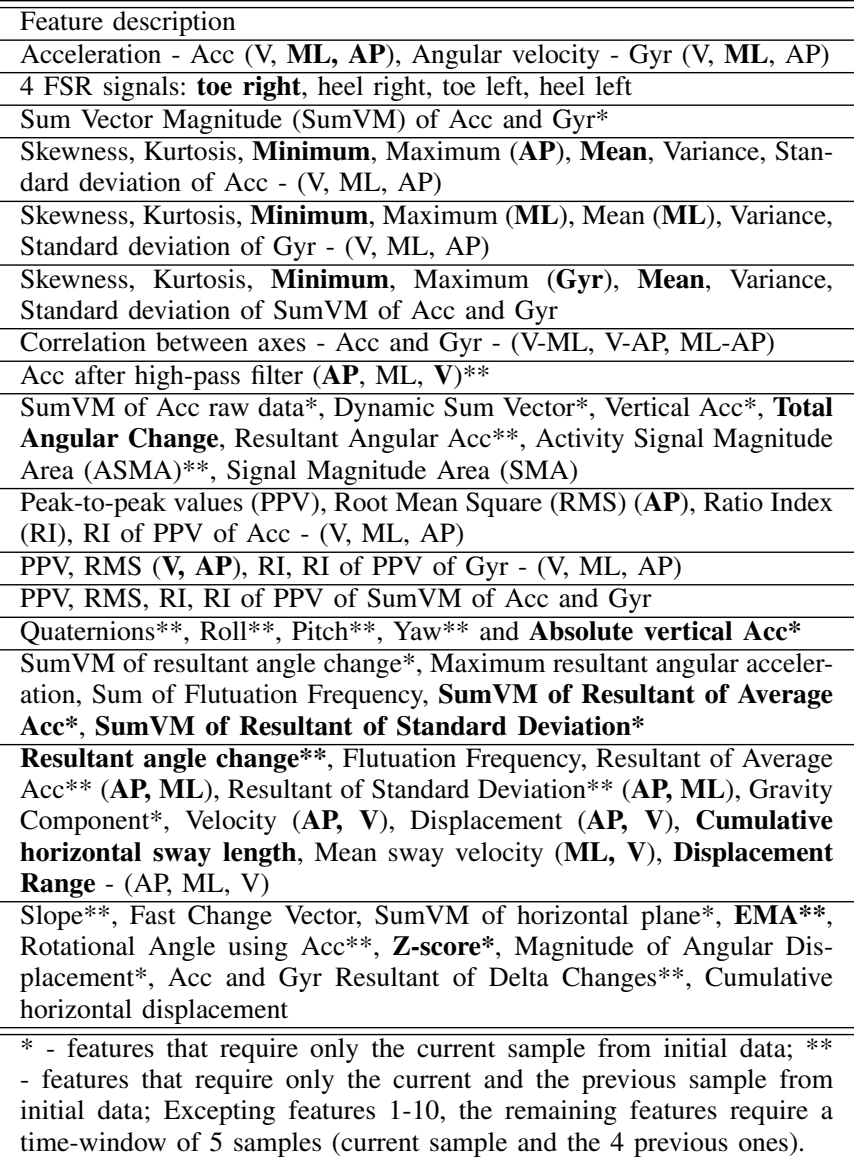

end-users. The proposed strategy is accurate (higher than $95 \%$ ) for a considerable sample of healthy subjects and detection times indicate a detection closer to the beginning of the near-fall than to the recovery stage. Future work focus on i) investigate the best IMU location and computational load of the proposed strategy; ii) use data from older adults to obtain results more related to end-users; and iii) use this information in a closed-loop system capable of actuating in high fall risk situations preventing the fall.

\section{REFERENCES}

[1] A. Pereira, N. F. Ribeiro, and C. P. Santos, "A survey of fall prevention systems implemented on smart walkers *," in 2019 IEEE 6th Portuguese Meeting on Bioengineering (ENBENG), pp. 1-4, Feb 2019.

[2] W. Xu, J. Huang, and L. Cheng, "A Novel Coordinated Motion FusionBased Walking-Aid Robot System," Sensors, vol. 18, no. 9, 2018.

[3] S. Taghvaei, Y. Hirata, and K. Kosuge, "Vision-based human state estimation to control an intelligent passive walker," in 2010 IEEE/SICE International Symposium on System Integration, pp. 146-151, 2010.

[4] S. Taghvaei and K. Kosuge, "Image-based fall detection and classification of a user with a walking support system," Frontiers of Mechanical Engineering, vol. 13, no. 3, pp. 427-441, 2018.

[5] I. Pang, Y. Okubo, D. Sturnieks, S. R. Lord, and M. A. Brodie, "Detection of Near Falls Using Wearable Devices: A Systematic Review," 2019.

[6] J. Figueiredo, P. Félix, L. Costa, J. C. Moreno, and C. P. Santos, "Gait event detection in controlled and real-life situations: Repeated measures from healthy subjects," IEEE Transactions on Neural Systems and Rehabilitation Engineering, vol. 26, pp. 1945-1956, Oct 2018. 